\title{
Contribution à l'étude de l'origine des levures du fromage de Camembert *
}

\author{
C Baroiller, JL Schmidt **
}

avec la collaboration technique de M Lapadu-Hargues

INRA, Paris-Grignon, laboratoire de recherches de la chaire de technologie, 78850 Thiverval-Grignon, France

(Reçu le 3 octobre 1989; accepté le 10 novembre 1989)

\begin{abstract}
Résumé - Des études antérieures sur la flore levure du fromage de Camembert ont montré que seuls 5 genres et espèces (Kluyveromyces marxianus var. lactis, Kl. marxianus var. marxianus, Debaryomyces hansenii, Saccharomyces cerevisiae et Zygosaccharomyces rouxii), et pour certaines leurs formes imparfaites, étaient prédominants. Il était, par conséquent, intéressant de connaître leur origine et de déterminer si la composition peu diversifiée de cette flore résultait de celle-ci ou était plutôt l'aboutissement d'un processus évolutif. Pour cela, 1576 souches ont été isolées à partir de prélèvements effectués à différents stades de la transformation du lait en fromage, dans un établissement industriel du Perche, chaque mois pendant une année. De plus, 220 souches ont été prélevées dans diverses exploitations agricoles livrant leur lait à cette entreprise. Une grille simplifiée d'identification, mise au point au laboratoire, a été utilisée. Une analyse statistique des résultats obtenus a montré que Kl. marxianus var. lactis et sa forme anascosporogène Candida sphaerica étaient prédominantes à tous les stades de la fabrication et que la relative diversité des espèces présentes dans le lait de collecte ne se retrouvait pas au niveau de la flore des fromages. Le processus technologique semble donc exercer un certain effet sélectif. L'identification, par des méthodes classiques, des souches isolées dans les exploitations agricoles, n'a pas révélé la présence marquante de l'une des 5 espèces principales de la flore levure du fromage.
\end{abstract}

levure / fromage / camembert / écologie microbienne

Summary - Study on the origins of yeasts from Camembert cheese. Previous studies on Camembert cheese yeasts showed that only 5 genera and species (Kluyveromyces marxianus var. lactis, KI. marxianus var. marxianus, Debaryomyces hansenii, Saccharomyces cerevisiae and Zygosaccharomyces rouxii) and only their anascosporogenous forms for some of them were predominant. It was then interesting to find out their origin to determine whether the little variation in composition of this yeast flora resulted from itself or was the outcome of a selective process. Consequently, 1576 strains were isolated from samples carried through the different stages of the processing of milk into cheese, within a Normandy dairy plant each month for one year. Moreover, 220 strains were sampled from different farms supplying the dairy plant with milk. A simplified identification scale, elaborated in our laboratory, was applied. Statistical analysis of the results showed that Kluyveromyces marxianus var. lactis and its anascosporogenous form Candida sphaerica were predomi-

* Etude inscrite dans le cadre d'une convention CEE ayant pour objet l'amélioration de la qualité du camembert et réalisée en collaboration avec les fromageries Lutin.

${ }^{*}$ Correspondance et tirés à part 
nant throughout all the cheese processing stages and that the relative diversity of the species in the collected milk did not appear in the cheese yeasts. Technological processing seems to have a selective action. The identification of the farms strains, with classical methods, showed no important presence of one of the 5 main cheese species.

yeast / cheese / camembert cheese / microbial ecology

\section{INTRODUCTION}

Les levures appartiennent à la flore microbienne normale de la plupart des fromages et, dans certains types de pâtes, elles sont susceptibles de participer activement aux modifications biochimiques qui sont à l'origine du développement de la saveur et de l'arôme (Gripon, 1978; Schmidt et al, 1979; Lenoir et al, 1983). Leur identification selon les méthodes conventionnelles (Lodder, 1971; Barnett et Pankhurst, 1974; Barnett et al, 1983; Kreger Van Rij, 1984) repose sur la détermination de divers caractères morphologiques ou physiologiques d'une mise en œuvre parfois lourde ou difficile.

Selon les données de Barnett et al (1983), 469 espèces de levures regroupées en 62 genres ont été recensées; or, seul un nombre restreint d'entre elles a été mis en évidence sur les différents types de fromages qui ont été étudiés:

- Devoyod et Sponem (1970), sur le fromage de Roquefort, observent, qu'avant salage, les levures présentes au centre du fromage sont essentiellement des espèces qui fermentent le lactose (Kluyveromyces marxianus var. lactis et sa forme imparfaite Candida sphaerica);

- Vergeade (1975) considère que Debaryomyces hansenii est l'espèce dominante de la flore levure normale du fromage de Saint-Nectaire, tant à l'intérieur qu'à la surface de la pâte;

- les études concernant le cantal (Millet et al, 1974), le Saint-Nectaire (Dale, 1972), le Cabrales (Nunez et al, 1981) et le Saint-Paulin (Ducastelle et Lenoir, 1965) ont reconnu la présence d'espèces apparcenant, pour l'essentiel, aux genres Kluyveromyces, Debaryomyces, Saccharomyces, Pichia, Hansenula, Rhodotorula et Candida (Choisy et al, 1987).

Les premiers travaux sur le fromage de Camembert sont relativement anciens (eg Guittonneau et al, 1939) et ils signalaient la présence de levures du genre Torula en surface. Lenoir et Auberger (1966) constatent que les levures présentes en surface, au nombre de $10^{9}$ UFC par gramme de pâte après une dizaine de jours d'affinage, rassemblent des espèces sporogènes proches du genre Saccharomyces et des levures asporogènes appartenant aux genres Candida et Rhodotorula. Les travaux de Schmidt et Lenoir $(1978 ; 1980)$ ont approfondi la connaissance de la flore levure du fromage de Camembert au lait cru en étudiant 5 fabrications différentes d'origine normande. Les espèces identifiées à partir d'une collection de près de 520 souches sont, pour l'essentiel, Kluyveromyces marxianus var. lactis et sa forme imparfaite ( $34 \%$ de la population), Kluyveromyces marxianus var. marxianus (15\%), Saccharomyces cerevisiae $(22 \%)$ et Debaryomyces hansenii (14\%). D'autres espèces ont été rencontrées seulement dans certains cas et elles peuvent être considérées comme mineures. Le fromage est donc un biotope dont la flore levure est relativement peu diversifiée; aussi, la suppression de tests d'identification qui concernent principalement des levures issues d'autres origines est-elle envisageable. Une simplification de la méthode 
d'identification permet alors de réaliser une étude sur l'origine des principales espèces de levures du fromage de Camembert, étude qui suppose l'isolement et la caractérisation d'un grand nombre de souches.

Le présent travail a pu être mené à bien grâce à l'utilisation de la grille simplifiée d'identification mise au point antérieurement (Baroiller et Schmidt, 1984; Baroiller, 1988). II est justifié par le fait, qu'à notre connaissance, aucune recherche ne paraît avoir porté sur l'origine des levures présentes dans les fromages. En revanche, d'autres écosystèmes ont été relativement bien étudiés. Tel est le cas, en œnologie, des raisins, des moûts et des vins pour lesquels les travaux de Mrak et McClung (1940) en Californie, de Renaud (1950) et de Brechot et al (1962) en Beaujolais, de Peynaud et Domercq (1953) et de Domercq (1957a et b) en Gironde ont montré l'existence d'une évolution de la flore au cours des différentes étapes de la vinification et que celle-ci était pratiquement indépendante de la nature des cépages. Les travaux de Castelli (eg 1967) ont mis clairement en évidence que la population en levures sporogènes augmente au fur et à mesure que l'on progresse vers le sud de I'Italie (Kloeckera est dominant au nord et le genre Hansenula l'est en dessous du 41e parallèle) et que l'altitude influait aussi sur cette répartition. D'autres travaux ont porté sur les levures de la région de Cognac (Park, 1974) et sur les levures du cidre (eg Michel et al, 1988). Certains écosystèmes comme les arbres et les cactées (eg Lachance et al, 1982), les eaux maritimes et fluviales (Morris, 1975; Hinzelin et Lectard, 1978), les mammifères et les oiseaux (Saez, 1979) ont été également analysés.

De toutes ces études, il ressort que les levures asporogènes appartenant aux genres Kloeckera, Candida et Rhodotorula ainsi que les genres sporogènes comme
Hanseniaspora, Torulaspora, Pichia, Saccharomyces et Hansenula sont constamment rencontrés, leur fréquence variant légèrement selon leurs origines.

\section{PROTOCOLE EXPÉRIMENTAL}

\section{L'étude a consisté :}

- dans un premier temps, à rechercher les principales espèces de levures rencontrées dans les fromages de l'entreprise aux différents stades de la fabrication en utilisant la grille simplifiée d'identification mise au point antérieurement (Baroiller et Schmidt, 1984).

- dans un second temps, à isoler des souches dans quelques exploitations agricoles de la zone de collecte en différents points de prélèvement. L'identification de ces souches a été conduite selon les concepts classiques décrits par Lodder (1971), Barnett et Pankhurst (1974), Barnett et al (1979; 1983) et Kreger Van Rij (1984). En effet, compte tenu de la diversité des genres et espèces de levures susceptibles d'être présents dans les écosystèmes étudiés (Phaff et al, 1978), il pouvait être présumé que la grille simplifiée s'avèrerait inadaptée.

\section{Isolement des souches}

\section{Stades de prélèvements}

Dans les exploitations agricoles

Les prélèvements ont été effectués en différents points susceptibles d'être à l'origine d'une contamination du lait en levures:

- l'alimentation des animaux : herbe, fourrage, ensilage et aliment de complément (concentré);

- les fèces des vaches laitières; 
- l'environnement : eau des abreuvoirs, atmosphère des salles de traite, sol (à une profondeur d'environ $5 \mathrm{~cm}$ ) considéré comme un réservoir naturel de ces microorganismes (Di Menna, 1978). Ces opérations ont été réalisées en avril.

\section{Dans l'entreprise fromagère}

Certains isolements ont eu lieu tous les mois, d'autres tous les 4 mois, ceci sur une période d'un an. Les prélèvements mensuels concernent :

- le lait cru réfrigéré, issu du mélange de 3 échantillons prélevés sur chacun des 3 tanks de stockage de l'entreprise (dénomination de l'échantillon : A);

- le lait de fabrication avant emprésurage (B);

- le caillé après salage issu du mélange de 3 pièces $(\mathrm{C})$;

- les fromages de 10 jours et en fin d'affinage (environ 35 jours), respectivement $J$ et $\mathrm{K}$, avec 2 prélèvements, l'un, issu du mélange de 3 pâtes fromagères (intérieur, $\mathrm{Jl}$ et KI), l'autre, du mélange des 3 surfaces (JS et KS), la séparation des parties internes et superficielles étant effectuée selon la technique décrite antérieurement (Schmidt et Lenoir, 1978);

- l'atmosphère des salles de fabrication, d'égouttage et des haloirs (échantillons D, E, F);

Tous les 4 mois, des souches ont également été isolées à partir :

- de caillés en cours d'égouttage (G) et de caillés avant salage $(H)$, toujours sur le mélange de 3 pièces;

- des bacs de saumurage (I).

Les prélèvements relatifs au lait et aux fromages aux différentes étapes concernent les mêmes fabrications.

\section{Méthodes d'isolement des souches}

Les prélèvements sont ensemencés dans des boîtes de Pétri sur un milieu sélectif pour levures et moisissures (Potato Dextrose Agar Difco $n^{\circ} 13, \mathrm{pH} \mathrm{3,5}=$ PDA). Le mode d'ensemencement varie selon l'origine des échantillons :

- pour l'air ambiant, il se fait par exposition directe de boîtes ouvertes à l'abri des turbulences pendant des intervalles de temps donnés : 1 - 5 - 10 et 30 min pour les salles de fabrication; 15 à 20 min pour les ambiances des salles de traite;

- les échantillons liquides (lait, saumure, eau des abreuvoirs) sont incorporés, après dilutions décimales, au milieu PDA;

- les caillés ou les fromages sont mis en suspension dans une solution stérile de citrate de sodium à $2 \%$ (Lenoir, 1962), puis après dilutions, l'ensemencement des boîtes est effectué comme précédemment;

- les échantillons solides, recueillis dans les exploitations à l'aide de spatules stériles et placés dans des erlenmeyers également stériles, sont passés à l'ultra-turrax $30 \mathrm{~s}$ à 16000 tours/min en présence de $100 \mathrm{ml}$ d'eau physiologique stérile (Piton, Richard, 1983). Les suspensions subissent ensuite une agitation sur table rotative durant 5 à $6 \mathrm{~h}$ à $25^{\circ} \mathrm{C}$ afin de disperser les levures qui adhèrent fortement à leurs substrats puis sont ensuite filtrées sur papier Whatman $n^{\circ} 42$ (Martini et al, 1980). Pour certains échantillons, des dilutions décimales successives sont pratiquées.

Dans tous les cas, le dénombrement des colonies est réalisé après 3 jours d'incubation à $25^{\circ} \mathrm{C}$. Seule l'évolution de la flore levure en fromagerie, au cours de l'année, est mentionnée dans le Tableau I. 
Tableau I. Evolution quantitative de la flore levure, au cours de l'année, en fromagerie (UFC par ml ou par g de pâte). Absence de prélèvement ou prélèvement effectué tous les 4 mois. A : lait cru réfrigéré; B : lait avant emprésurage; C : caillé avant salage; $\mathrm{Jl}$ : fromages $10 \mathrm{j}$, intérieur; $\mathrm{KI}$ : fromages $35 \mathrm{j}$, intérieur; JS : fromages $10 \mathrm{j}$, surface; KS : fromages $35 \mathrm{j}$, surface; D, E, F : atmosphères des salles de fabrication, d'égouttage et des haloirs; $G$ : caillés en cours d'égouttage; $\mathrm{H}$ : caillés avant salage; I : bacs de saumurage.

Quantitative evolution of yeast flora during the year in cheese-making (CFU) per $\mathrm{ml}$ or per $\mathrm{g}$ of cheese).

\begin{tabular}{|c|c|c|c|c|c|c|c|c|c|}
\hline \multicolumn{10}{|c|}{ Stade } \\
\hline & $A$ & $B$ & $G$ & $H$ & $C$ & JS & ال & $K S$ & $K I$ \\
\hline \multicolumn{10}{|l|}{ Mois } \\
\hline Nov. & $2,8.10^{4}$ & $3,1.10^{3}$ & $1,1.10^{4}$ & $1,1.10^{5}$ & $1,1.10^{5}$ & $1,2.10^{7}$ & $1,0.10^{5}$ & $3,3.10^{8}$ & $2,8.10^{5}$ \\
\hline Déc. & $1,2.10^{5}$ & $2,6.10^{3}$ & - & - & $6,5.10^{4}$ & $1,1.10^{7}$ & $2,6.10^{5}$ & - & - \\
\hline Janv. & $1,1.10^{3}$ & $1,1.10^{4}$ & - & - & $1,9.10^{5}$ & $7,5.10^{6}$ & $1,0.10^{5}$ & $4,2.10^{7}$ & $1,1.10^{6}$ \\
\hline Fév. & $1,5.10^{4}$ & - & - & - & $4,0.10^{4}$ & $5,6.10^{7}$ & $6,3.10^{5}$ & $6,2.10^{4}$ & $5,3.10^{4}$ \\
\hline Mars & $1,0.10^{4}$ & $1,2.10^{4}$ & $1,4.10^{4}$ & $2,5.10^{5}$ & $7,1.10^{5}$ & $9,1.10^{7}$ & $1,7.10^{6}$ & $1,6.10^{7}$ & $8,1.10^{3}$ \\
\hline Avr. & $1,4.10^{4}$ & $3,1.10^{3}$ & - & - & $1,8.10^{5}$ & $2,7.10^{7}$ & $2,7.10^{5}$ & $5,6.10^{7}$ & $4,3.10^{6}$ \\
\hline Mai & $3,0.10^{4}$ & $1,3.10^{4}$ & - & - & $1,3.10^{5}$ & $3,3.10^{7}$ & $3,4.10^{5}$ & $5,3.10^{8}$ & $7,6.10^{4}$ \\
\hline Juin & $1,5.10^{4}$ & $1,7.10^{4}$ & - & - & $3,7.10^{5}$ & $3,2.10^{7}$ & $2,5.10^{5}$ & - & - \\
\hline Juillet & $4,6.10^{4}$ & $1,3.10^{4}$ & - & - & $2,1.10^{5}$ & - & - & $5,4.10^{6}$ & $2,2.10^{5}$ \\
\hline Août & $1,9.10^{4}$ & $1,3.10^{4}$ & $1,1.10^{4}$ & $4,1.10^{4}$ & $6,0.10^{4}$ & - & $3,9.10^{4}$ & $5,5.10^{6}$ & $3,0.10^{4}$ \\
\hline Sept. & - & - & - & - & - & - & - & $4,2.10^{7}$ & $3,5.10^{4}$ \\
\hline Oct. & $3,5.10^{3}$ & $1,4.10^{4}$ & $1,8.10^{5}$ & $2,3.10^{5}$ & $2,2.10^{5}$ & $2,1.10^{8}$ & $1,4.10^{5}$ & - & - \\
\hline Moyenne & $2,7.10^{4}$ & $1,0.10^{4}$ & $5,4.10^{4}$ & $1,6.10^{5}$ & $2,0.10^{5}$ & $5,0.10^{7}$ & $4,2.10^{5}$ & $1,1.10^{8}$ & $6,8.10^{5}$ \\
\hline
\end{tabular}




\section{Purification et conservation des souches}

A partir de chaque boîte d'isolement contenant une centaine de colonies, 5 à 10 colonies sont prélevées au hasard (eg Schmidt et Lenoir, 1978) et ensuite repiquées en milieu liquide non sélectif, le Yeast Extract Glucose (YEG) (Vergeade, 1975). Après incubation 3 jours à $25^{\circ} \mathrm{C}$, il est procédé à un nouvel isolement sur PDA. Puis, dans des conditions identiques de développement, une colonie est transplantée en YEG. A ce stade, la pureté des souches est contrôlée au microscope. $\mathrm{Si}$ une souche paraît contaminée (moisissure, Bacillus...) ou impure, elle subit un nouveau cycle de purification. Les souches sont alors ensemencées sur gélose inclinée à base de YEG et, après développement, conservées à $4^{\circ} \mathrm{C}$.

Aux différents points de prélèvements de la fromagerie, 1475 souches ont été ainsi isolées entre les mois de novembre et d'octobre. En février et mars, des prélèvements complémentaires au niveau des saumures ont permis d'isoler 101 souches. La population issue de la fromagerie représente donc au total 1576 souches. Leur répartition par mois et par stade de prélèvement est présentée dans le tableau II.

Dans les exploitations agricoles, 220 souches ont été isolées; leur répartition par type de prélèvement est la suivante : herbe, 31 souches, fourrage, 19; ensilage, 31 ; concentré, 15; eau des abreuvoirs, 44; fèces, 44; sol, 14 (tableau HI).

\section{Identification des souches}

La méthode simplifiée, utilisée pour l'identification des souches issues des prélève- ments effectués en fromagerie, repose sur I'utilisation de divers tests morphologiques et biochimiques (Baroiller et Schmidt, 1984). Les souches provenant des exploitations agricoles ont été identifiées selon les méthodes classiques; cependant, les épreuves de croissance en milieu aérobie ont été réalisées sur milieu solide à l'aide d'un ensemenceur «Multipoint" (RidgwayWatt, 1979).

Les différentes épreuves sont les suivantes :

- épreuves morphologiques :

- aspects culturaux en YEG liquide et sur YEG gélosé;

- aspect cellulaire (forme et taille des cellules, mode de reproduction végétative);

- formation d'ascospores sur 2 milieux différents afin de répondre aux exigences parfois opposées de ces microorganismes : un milieu riche ( $\mathrm{V} 8$, Wickerham, 1946) (jus de légumes V 8 , Campbell Soup Company) et un milieu pauvre (Fowell, 1952). Le nombre et la forme des ascospores éventuellement présentes sont notés après incubation 15 jours à $25^{\circ} \mathrm{C}$;

- aptitude à la filamentisation (formation éventuelle de pseudomycelium) sur maïs agar (Bernhardt, 1946);

- épreuves biochimiques :

- fermentation de 7 glucides selon la méthode en milieu liquide (Lodder, 1971);

- assimilation de composés azotés ou carbonés. Dans le premier cas, le milieu utilisé est le Yeast Carbon Base (Difco $n^{\circ}$ 391) (Wickerham, 1946); dans le second cas, le milieu Yeast Nitrogen Base (Difco $n^{\circ}$ 392) (Wickerham et Burton, 1948), selon le protocole décrit par Baroiller et Schmidt (1984);

- résistance à un antibiotique, la cycloheximide à $0,01 \%$, où les réponses sont de type «tout ou rien". 
Tableau II. Répartition des prélèvements effectués dans la fromagerie selon les stades et les mois (en nombre de souches). ${ }^{\circ}$ La signification des lettres est précisée dans le tableau I; " prélèvements réalisés ultérieurement; absence de prélèvement ou prélèvement effectué tous les 4 mois.

Breakdown of samples from cheese factories according to stage of maturity and month (number of strains).

\begin{tabular}{|c|c|c|c|c|c|c|c|c|c|c|c|c|c|}
\hline & \multicolumn{13}{|c|}{ Points de prélèvements ${ }^{\circ}$} \\
\hline & \multirow[b]{2}{*}{$A$} & \multirow[b]{2}{*}{$B$} & \multirow[b]{2}{*}{$G$} & \multirow[b]{2}{*}{$H$} & \multirow[b]{2}{*}{$c$} & \multicolumn{2}{|c|}{$J$} & \multicolumn{2}{|c|}{$K$} & \multicolumn{4}{|c|}{ Atmosphères } \\
\hline & & & & & & $s$ & 1 & $s$ & 1 & 1 & $D$ & $E$ & $F$ \\
\hline \multicolumn{14}{|l|}{ Mois } \\
\hline Nov. & 15 & 18 & 11 & 13 & 11 & 7 & 7 & 10 & 8 & 1 & 16 & 7 & - \\
\hline Déc. & 7 & 17 & - & - & 8 & 11 & 11 & - & - & - & 10 & - & 8 \\
\hline Janv. & 18 & 12 & - & - & 11 & 10 & 11 & 8 & 15 & 一 & 9 & 17 & 1 \\
\hline Fév. & 19 & 10 & - & - & 16 & 11 & 8 & 15 & 11 & 一 & * 18 & 9 & 7 \\
\hline Mars & 25 & 27 & 21 & 21 & 17 & 14 & 11 & 8 & 12 & 6 & 10 & 7 & 10 \\
\hline Avr. & 24 & 30 & - & - & 13 & 10 & 13 & 14 & 10 & - & 15 & 2 & 1 \\
\hline Mai & 36 & 35 & - & - & 12 & 20 & 12 & 9 & 9 & - & 9 & 6 & 2 \\
\hline Juin & 19 & 23 & - & - & 8 & 10 & 15 & 10 & 9 & - & 8 & 6 & 3 \\
\hline Juillet & 56 & 17 & - & - & 22 & - & - & 9 & 9 & - & 13 & - & 2 \\
\hline Août & 15 & 18 & 15 & 5 & 19 & 13 & 9 & 8 & 8 & 1 & - & 8 & - \\
\hline Sept. & 10 & 20 & - & - & 13 & 6 & 4 & 13 & 13 & - & - & 11 & - \\
\hline Oct. & 7 & 25 & 16 & 13 & 18 & 11 & 14 & - & - & 1 & 11 & 1 & 1 \\
\hline Total $=1576$ & 251 & 252 & 63 & 52 & 168 & 122 & 122 & 104 & 104 & 110 & 119 & 74 & 35 \\
\hline
\end{tabular}


Tableau III. Répartition des levures identifiées dans les exploitations agricoles selon la nature du prélèvement et les sous-classes de levures (en nombre de souches). Souches identifiées : 204; Fungi imperfecti : $60 \%$; Ascomycètes : $36 \%$; * Ambiance des salles de traite.

Breakdown of identified yeasts in farms according to sampling nature and different species of yeasts (number of strains).

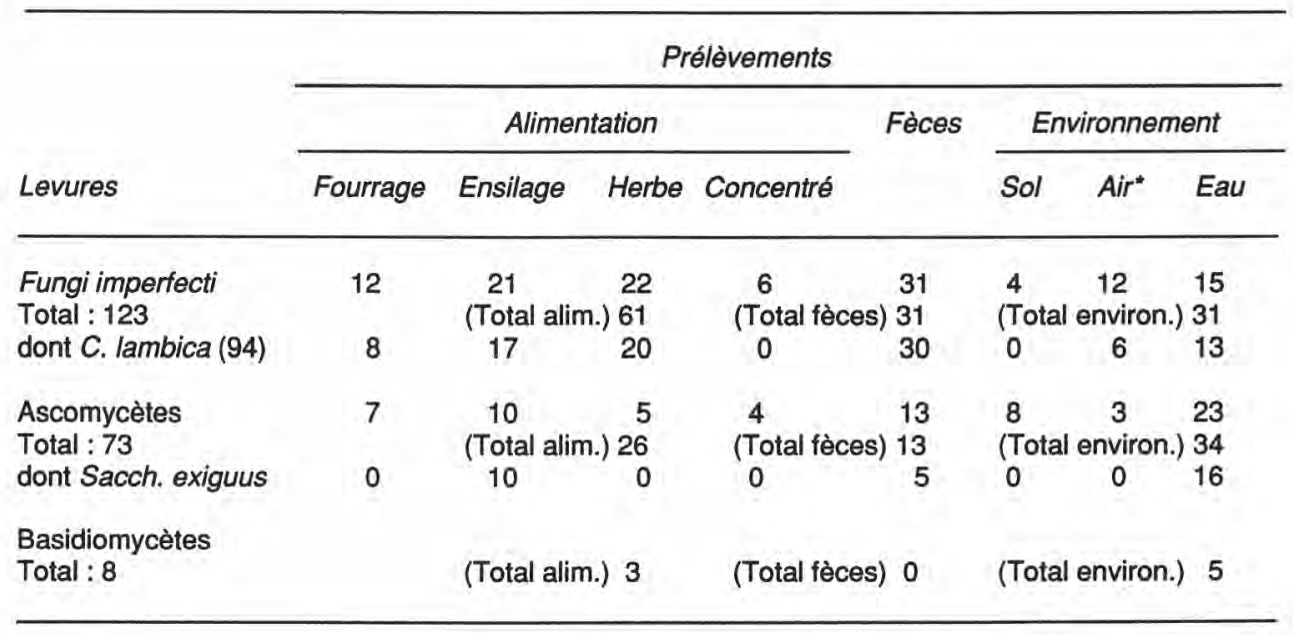

\section{Traitement statistique des données}

Etant donné le nombre important de souches isolées et identifiées, pour apprécier l'évolution des genres et des espèces en fonction des stades de prélèvements et des périodes de l'année, un traitement statistique des résultats s'imposait. Le modèle retenu, le mieux adapté à l'analyse de ces résultats, est le modèle logarithme linéaire. (Daudin, communication personnelle).

\section{RÉSULTATS ET DISCUSSION}

Les différents prélèvements réalisés ont permis l'acquisition de 2 catégories d'informations : des indications quantitatives sur l'importance des populations, des données qualitatives concernant l'identité des levures présentes.

\section{Importance des populations}

Sur 3 des 4 points de prélèvements (fèces, eau et sol), il existe, selon les exploitations agricoles étudiées, une certaine homogénéité dans les résultats :

— fèces : $2,5.10^{3}$ à $5,8.10^{4}$ levures/g;

— sol : $4.10^{2}$ à $4.10^{3}$ levures/g;

— eau : 13 à 38 levures/ml.

Il est intéressant d'observer que pour les prélèvements d'eau, les populations les plus fortes correspondent à des prises d'échantillons en eau stagnante. En ce qui concerne les ensilages, les variations de peuplement en levures, selon les exploitations, sont très grandes puisqu'elles oscillent de moins de $10^{2}$ à $1,6 \cdot 10^{7} \mathrm{UFC} / \mathrm{g}$. Les chiffres obtenus dans les eaux et les sols concordent avec ceux mentionnés dans la littérature (Di Menna, 1978; Phaff et al, 1978). Pour les ensilages, les popu- 
lations que nous avons trouvées sont en accord avec les observations de Engel (1986) qui fait également état d'une très grande dispersion des résultats. En revanche, pour les fèces bovines, ce qui est d'ailleurs plutôt surprenant, aucune donnée de référence n'est, à notre connaissance, bien établie.

Les résultats obtenus en fromagerie (tableau I) montrent que la contamination initiale en levures dans le lait de collecte (A) peut présenter, selon les mois, des fluctuations notables : un maximum est observé au mois de décembre $\left(10^{5}\right)$ et un minimum en janvier et octobre $\left(10^{3}\right)$; les autres mois, la population se situe aux environs de $10^{4}$ UFC/ml. Dans le lait de fabrication (B), le peuplement en levures apparaît plus stable, son niveau étant voisin de celui des laits de collecte (environ $10^{4} \mathrm{UFC} / \mathrm{ml}$ ). Ces chiffres ne concordent pas avec ceux mentionnés par Devoyod et Sponem (1970) qui ont dénombré de $10^{2}$ à $10^{3} \mathrm{UFC} / \mathrm{ml}$ dans le lait de brebis destiné à l'élaboration du fromage de Roquefort. En revanche, nos résultats sont assez proches de ceux de Millet et al (1974) qui ont mis en évidence, dans le lait entrant dans la fabrication du Cantal, au moment de l'emprésurage, une population de $10^{4} \mathrm{UFC} / \mathrm{ml}$ ou de ceux enregistrés par Engel (1986) sur des laits crus destinés à la fabrication de Camemberts allemands. Dans les caillés, avant salage ( $G$ et $H)$, les levures sont en nombres assez réguliers $\left(10^{4}\right.$ à $10^{5}$ UFC/ g). Le salage semble peu affecter la densité de population puisque les proportions restent comparables après saumurage $(C)$.

En ce qui concerne les fromages affinés ou en cours d'affinage, des fluctuations importantes sont observées, notamment sur les échantillons les plus âgés :

- en surface des fromages, à 10 jours (JS), $7.10^{6}$ à $2.10^{8} \mathrm{UFC} / \mathrm{g}$, à 35 jours (KS), $6.10^{4}$ à $5.10^{8} \mathrm{UFC} / \mathrm{g}$;
- à l'intérieur des fromages, à 10 jours (JI), $4.10^{4}$ à $2.10^{6} \mathrm{UFC} / \mathrm{g}$, à 35 jours (KI), $8.10^{3}$ à $6.10^{6} \mathrm{UFC} / \mathrm{g}$.

II apparaît, comme cela avait déjà été montré par Schmidt et Lenoir (1978; 1980), que la flore levure est beaucoup plus importante à la surface des fromages qu'à l'intérieur de la pâte. L'écart de population relevé entre les 2 parties (100/1) est du même ordre de grandeur que celui mis en évidence sur les fromages de lait cru; en revanche, le maximum de population à 10 jours est, pour les Camemberts étudiés (2.108 UFC/g), sensiblement inférieur à celui observé antérieurement $\left(5.10^{8}\right.$ à $\left.3.10^{9} \mathrm{UFC} / \mathrm{g}\right)$.

En outre, si pour les fabrications de fromages au lait cru le peuplement maximum à 10 jours était suivi d'une diminution du nombre de cellules, il n'en est pas de même dans le cas présent où la densité cellulaire semble se stabiliser entre 10 et 35 jours, voire même progresser.

\section{Identité des espèces de levures pré- sentes}

\section{Flore des exploitations agricoles}

Sur les 220 souches isolées, pour l'ensemble des prélèvements effectués dans les exploitations agricoles, 204 ont pu être identifiées. Les levures anascosporogènes représentent $60 \%$ de la population (123 souches) et les levures ascosporogènes $36 \%$ (73 souches). Huit souches appartiennent à la sous-classe des Basidiomycètes (tableau III).

Au sein des levures ne formant pas de spores, 10 espèces ont été recensées contre 16 espèces productrices de spores. Ainsi, la diversité est plus grande pour les levures sporogènes. Dans la population étudiée, 2 espèces sont particulièrement 
bien représentées et constituent, à elles seules, $60 \%$ de la flore: Candida lambica: 94 souches et Saccharomyces exiguus : 31 souches.

Ces espèces sont principalement isolées des fèces, des eaux d'alimentation et des ensilages, cela dans les 4 exploitations. En ce qui concerne les ensilages, nos résultats sont en accord avec ceux obtenus par Engel (1986) qui fait état de la présence dominante de l'espèce Candida lambica. La prédominance, dans la nature, des levures anascosporogènes, généralement admise (eg Phaff et al, 1978), se trouve, dans le cas présent, largement vérifiée. L'identité des autres espèces de levures isolées dans l'alimentation des animaux, les fèces et l'environnement des exploitations permet de faire 2 observations intéressantes :

- d'une part, les espèces rencontrées dans l'alimentation des animaux et dans les eaux des abreuvoirs sont également présentes dans les fèces : l'appareil digestif de l'animal n'apporterait donc pas de modifications majeures dans la nature et l'équilibre de la flore;

- d'autre part, parmi les espèces dominantes de la flore des fromages de $\mathrm{Ca}$ membert (Schmidt et Lenoir, 1978; 1980), seules quelques souches de Debaryomyces hansenii, Saccharomyces cerevisiae et Kluyveromyces marxianus var. marxianus ont été rencontrées aux différents points de prélèvements. En revanche, Kluyveromyces marxianus var. lactis et sa forme imparfaite Candida sphaerica, qui sont pourtant trouvées en grand nombre dans les fromages, n'ont jamais été mises en évidence.

Certaines hypothèses peuvent être avancées : le nombre de souches isolées est insuffisant certains points de prélèvements, sources possibles de contamination, n'ont pas été retenus (par exemple, les poils et la peau des animaux, la surface de la mamelle...), Kluyveromyces marxianus var. lactis est bien présente mais elle est trop largement dominée par les autres espèces pour pouvoir être isolée dans les conditions expérimentales adoptées.

\section{Flore des fromages et de la fromagerie}

Les résultats obtenus en fromagerie (tableaux IV et V) font apparaître 2 faits marquants : la prédominance de l'espèce Kluyveromyces marxianus var. lactis et de sa forme imparfaite Candida sphaerica, et la difficulté d'analyser la répartition des espèces ou des genres par mois et par stade, compte tenu des variations de la taille des échantillons prélevés et, par conséquent, la nécessité de dégager par un traitement statistique approprié la signification de ces résultats.

Les variations observées entre les différents mois, pour un même stade, semblent peu tributaires des saisons. En revanche, selon les étapes de la fabrication des fromages, il est possible de faire plusieurs constatations.

- II apparaît que dans le lait de collecte (A) et dans le lait avant emprésurage (B) un assez grand nombre de souches n'a pu être identifié avec la grille simplifiée : sur 404 souches issues de ces 2 stades de prélèvements, 94 ne possèdent pas un profil type; cependant, 43 souches présentent les mêmes réponses biochimiques. Elles ont été identifiées par la méthode classique et se répartissent de la façon suivante : Zygosaccharomyces bailii (20 souches), Zygosaccharomyces bisporus (13), espèce taxonomiquement très proche de la précédente, Yarrowia lipolytica (6), espèce déjà rencontrée dans les fromages de Saint-Nectaire (Dale, 1972), Candida cantarellii (4).

Elles sont regroupées sous l'appellation ZYC. Les autres souches notées X présentent des profils biochimiques très dissemblables et elles n'ont pu être identifiées. Ces souches des groupes ZYC et $X$ ont été, en majeure partie, isolées à partir du lait et des ambiances des salles de fabrica- 
Tableau IV. Répartition des levures identifiées en fromagerie en fonction du mois de prélèvement (en nombre de souches).

Breakdown of identified yeasts in cheesemaking, in terms of month of sampling (number of strains).

\begin{tabular}{|c|c|c|c|c|c|c|c|c|c|c|c|c|c|}
\hline \multicolumn{14}{|c|}{ Mois } \\
\hline & Nov & Déc & Janv & Fév & Mars & Avr & Mai & Juin & Juillet & Août & Sept. & Oct. & Total \\
\hline \multicolumn{14}{|l|}{ Espèces } \\
\hline Km. v. I. & 46 & 17 & 21 & 25 & 30 & 29 & 60 & 27 & 56 & 36 & 23 & 30 & 400 \\
\hline C. sph. & 31 & 14 & 40 & 37 & 43 & 39 & 32 & 22 & 39 & 21 & 19 & 30 & 367 \\
\hline Z. rouxii & 5 & 5 & 5 & 24 & 24 & 8 & 7 & 9 & 3 & 18 & 1 & 7 & 116 \\
\hline C. mogii & 6 & 4 & 12 & 8 & 30 & 8 & 2 & 9 & 4 & 6 & 2 & 3 & 94 \\
\hline D. hans. & 3 & 7 & 4 & 4 & 13 & 4 & 21 & 5 & 7 & 3 & 5 & 9 & 85 \\
\hline$K m$. v. $m$. & 0 & 1 & 0 & 9 & 2 & 3 & 0 & 7 & 2 & 6 & 28 & 6 & 64 \\
\hline C. kefyr. & 0 & 2 & 0 & 1 & 0 & 1 & 1 & 1 & 5 & 0 & 2 & 5 & 18 \\
\hline$K m$. v. b. & 8 & 7 & 4 & 11 & 7 & 0 & 8 & 4 & 1 & 1 & 0 & 0 & 51 \\
\hline Sac. cer. & 12 & 1 & 1 & 2 & 1 & 3 & 0 & 8 & 2 & 11 & 1 & 5 & 47 \\
\hline C. versat. & 3 & 1 & 0 & 0 & 0 & 1 & 0 & 0 & 0 & 0 & 1 & 0 & 6 \\
\hline Groupe ZYC & 6 & 9 & 18 & 7 & 21 & 24 & 11 & 5 & 7 & 6 & 2 & 17 & 133 \\
\hline $\begin{array}{l}\text { Groupe X } \\
\text { pigmt. }\end{array}$ & 1 & 0 & 1 & 1 & 0 & 1 & 1 & 0 & 0 & 3 & 1 & 0 & 9 \\
\hline Groupe X & 3 & 4 & 6 & 1 & 18 & 11 & 7 & 14 & 2 & 8 & 5 & 6 & 85 \\
\hline Total & 124 & 72 & 112 & 130 & 189 & 132 & 150 & 111 & 128 & 119 & 90 & 118 & 1475 \\
\hline
\end{tabular}


Tableau V. Répartition des levures identifiées en fromagerie en fonction du stade de prélèvement (en nombre de souches). ${ }^{\circ}$ Chiffres ne tenant pas compte du prélèvement réalisé ultérieurement; ${ }^{*}, A, B, \ldots$ : se reporter au tableau $I$.

Breakdown of identified yeasts in cheesemaling in terms of sampling (number of strains).

Stades de prélèvement *

$\begin{array}{lllllllllllllll}A & B & G & H & C & J S & J l & K S & K l & 1^{\circ} & D & E & F & T \text { Total }\end{array}$

\section{Espèces de levures}

Km. var. lactis

Cand. sphaerica

Z. rouxii

C. mogii

Deb. hansenii

$\mathrm{Km}$. var. marxianus

Cand. kefyr

$K m$. var. bulgaricus

Sac. cerevisiae

Cand. versatilis

Groupe ZYC

Groupe $X$ pigmenté

Groupe $X$

$\begin{array}{rrrrr}68 & 60 & 17 & 11 & 60 \\ 67 & 61 & 9 & 18 & 44 \\ 7 & 9 & 6 & 5 & 14 \\ 13 & 14 & 5 & 9 & 18 \\ 13 & 31 & 0 & 0 & 4 \\ 6 & 9 & 5 & 5 & 7 \\ 6 & 2 & 0 & 0 & 2 \\ 17 & 14 & 3 & 1 & 5 \\ 7 & 3 & 5 & 1 & 3 \\ 0 & 2 & 0 & 0 & 0 \\ 16 & 27 & 12 & 1 & 6 \\ 3 & 2 & 0 & 0 & 0 \\ 28 & 18 & 1 & 1 & 5\end{array}$

42
26
23
9
6
0
1
2
1
1
5
1
5

$\begin{array}{rrrr}26 & 31 & 28 & 0 \\ 34 & 26 & 34 & 2 \\ 11 & 17 & 5 & 0 \\ 5 & 6 & 6 & 3 \\ 5 & 1 & 0 & 2 \\ 8 & 6 & 18 & 0 \\ 6 & 1 & 0 & 0 \\ 2 & 3 & 3 & 1 \\ 19 & 0 & 7 & 0 \\ 0 & 0 & 0 & 0 \\ 3 & 9 & 2 & 1 \\ 0 & 0 & 0 & 0 \\ 3 & 4 & 1 & 0\end{array}$

$\begin{array}{rrrr}30 & 17 & 10 & 400 \\ 25 & 19 & 2 & 367 \\ 9 & 6 & 4 & 116 \\ 2 & 4 & 0 & 94 \\ 10 & 2 & 11 & 85 \\ 0 & 0 & 0 & 64 \\ 0 & 0 & 0 & 18 \\ 0 & 0 & 0 & 51 \\ 1 & 0 & 0 & 47 \\ 3 & 0 & 0 & 6 \\ 32 & 15 & 4 & 133 \\ 1 & 1 & 1 & 9 \\ 6 & 10 & 3 & 85\end{array}$


tions; ainsi, $76 \%$ des souches $X$ et $80 \%$ des souches ZYC ont de telles origines. Au niveau des fromages, lorsqu'elles sont présentes, elles le sont essentiellement en surface. Cette observation est intéressante car, à notre connaissance, il n'a jamais été signalé la présence d'une flore aussi diversifiée sur les autres types de pâtes fromagères étudiées.

- En comparant ces résultats avec ceux de Schmidt et Lenoir $(1978 ; 1980)$, il est

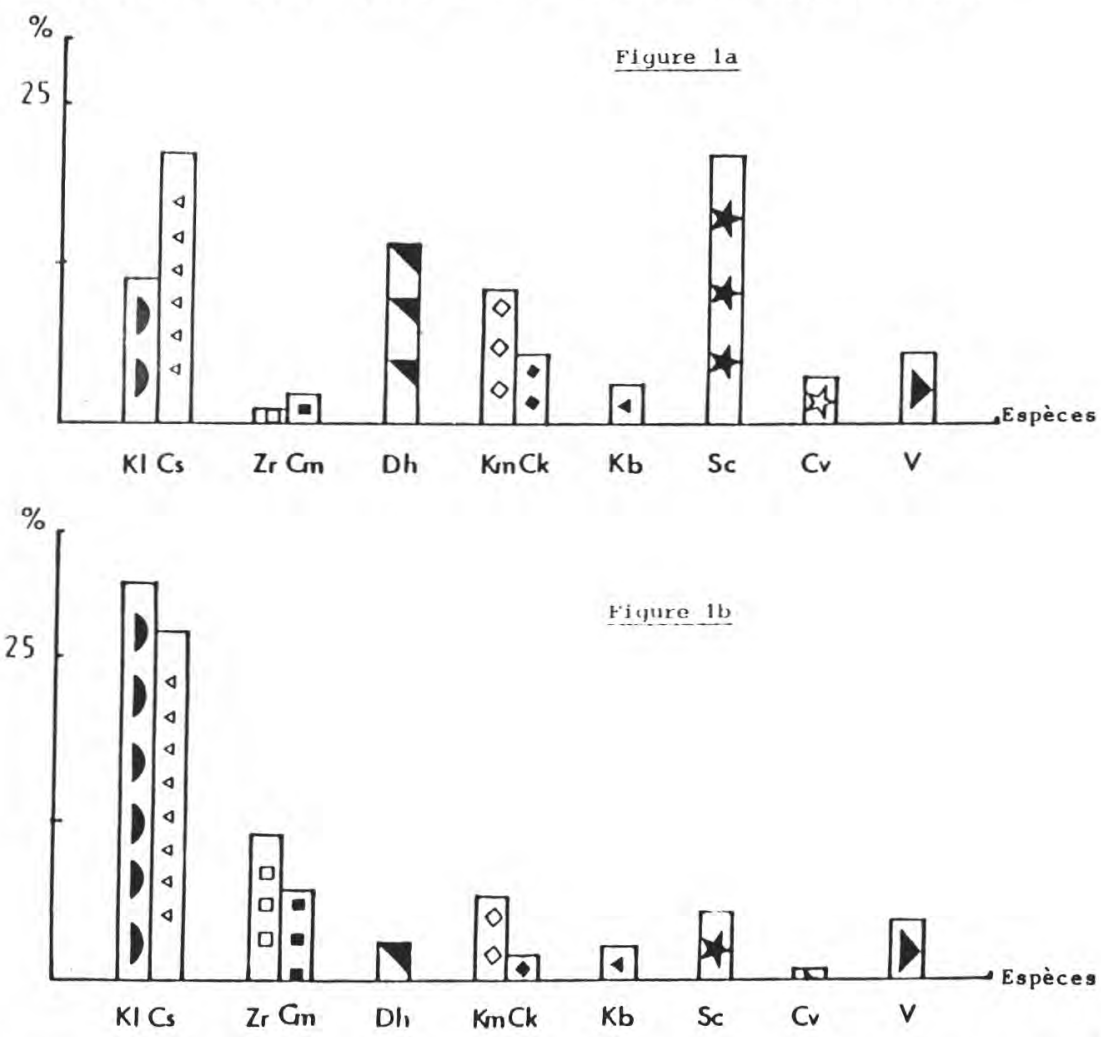

Fig. 1. Différence de composition de la flore levure du fromage de Camembert affiné selon les études réalisées (en \% du nombre de souches identifiées). Different composition of Camembert cheese yeast flora ripening according to recent studies (\% of identified number of strains). 1a : Résultats moyens obtenus sur 5 fabrications traditionnelles au lait cru (Travaux de Schmidt et Lenoir $(1978 ; 1980)$. Studies of Schmidt and Lenoir (1978-1980); mean results of 5 traditional cheesemaking methods with raw milk. 1b : Résultats moyens obtenus, dans la présente étude, sur une année. Mean results, in this current study, over a one-year period. Espèces représentées : KI : Kluyveromyces marxianus var. lactis, Cs : Candida sphaerica, $\mathrm{Zr}$ : Zygosaccharomyces rouxii, $\mathrm{Cm}$ : Candida mogii, Dh : Debaryomyces hansenii, $\mathrm{Km}$ : Kluyveromyces marxianus var. marxianus, $\mathrm{Ck}$ : Candida kefyr, $\mathrm{Kb}$ : Kluyveromyces marxianus var. bulgaricus, Sc : Saccharomyces cerevisiae, Cv : Candida versatilis, V : Autres espèces. possible de relever que la flore dominante reconnue par ces auteurs était principalement constituée par l'espèce Kluyveromyces marxianus var. lactis et sa forme imparfaite Candida sphaerica. Dans l'étude présente, la même observation peut être faite, ces espèces étant encore plus fortement représentées, alors que les espèces Saccharomyces cerevisiae, Candida versatilis et Debaryomyces hansenii apparaissent moins fréquentes (fig 1 ). 
- Les deux espèces Candida versatilis et Debaryomyces hansenii sont des levures halotolérantes et il est possible que le niveau de leur présence soit conditionné par le mode de salage, salage à sec en fabrication traditionnelle, salage en saumure dans le cas présent. Les prélèvements réalisés sur les saumures montrent qu'elles sont effectivement présentes bien qu'elles soient peu nombreuses dans les fromages.

\section{Traitement statistique des résultats}

D'un point de vue statistique, les résultats à analyser constituent une table de contingence multidimensionnelle; en effet, une table de contingence à $n$ dimensions correspond au classement d'un échantillon extrait d'une population donnée selon $n$ critères de classification (Collombier, 1976). La table de contingence considérée ici est à 3 dimensions qui, dans l'ordre du traitement informatique des données, sont les 3 variables suivantes : le stade de prélèvement, le mois de prélèvement, le genre ou l'espèce de levure concerné.

\section{Choix du programme}

Plusieurs modèles mathématiques peuvent assurer l'analyse, par traitement informatique, des tables de contingence multidimensionnelles. II est apparu que le modèle logarithme linéaire était le mieux adapté à la table de contingence obtenue (Daudin, communication personnelle). Ce modèle permet, en effet, de mettre en évidence, si elles existent, des relations significatives entre les variables prises 2 à 2 :

$$
\begin{aligned}
\log \left(n_{1.2 .3}\right)= & \chi+\psi_{1}+\beta_{2}+\gamma_{3}+\phi_{1.2}+\zeta_{1.3} \\
& +\varepsilon_{2.3}+v_{1.2 .3}
\end{aligned}
$$

où $1,2,3$, sont les variables précédemment définies, 1 et 2 étant les variables facteurs et 3 la variable réponse. $\psi$ met en évidence l'effet de la variable 1 sur l'ensemble de données $\beta_{2}$ et $\gamma_{3}$, de même, pour les variables 2 et $3 ; \phi_{1.2}$ détermine l'interaction qui existe ou non entre la variable "stade" et la variable "mois"; $\zeta_{1.3}$ entre le "stade" et "l'espèce"; $\varepsilon_{2.3}$ entre le "mois" et «l'espèce"; $v_{1.2 .3}$ pour l'essentiel des 3 variables.

Dans l'analyse statistique, 8 stades de prélèvements ont été pris en considération : A (lait de collecte), B (lait avant emprésurage), C (caillé après salage), JS et $\mathrm{JI}$ (fromages de 10 jours, en surface et à l'intérieur), KS et KI (fromages de 35 jours, en surface et à l'intérieur) et $D$ (atmosphère des salles de fabrication). La taille de la table de contingence à analyser est donc de 12 lignes (12 mois) $\times 8$ colonnes ( 8 stades) $\times 13$ espèces.

\section{Résultats}

Le test de la variable "espèce» est très significatif; en effet, les espèces ne sont pas présentes à la même fréquence selon les stades et les mois.

Le test du "mois» par rapport à "l'espèce" et celui du "stade" par rapport à "l'espèce" sont également hautement significatifs. Par conséquent, l'étude des modèles $1.2+1.3+2.3$ est justifiée et a permis de faire apparaître les résultats suivants :

- l'espèce Kluyveromyces marxianus var. lactis et sa forme imparfaite Candida sphaerica dominent significativement l'ensemble des données de la table de contingence. A un niveau moindre, Zygosaccharomyces rouxii et les espèces du groupe ZYC sont sensiblement plus représentées que les espèces restantes; 
- la considération de la variable "stade" (1) par rapport à la variable "espèce" (3) montre que le groupe $Z Y C$ et les souches $X$ (non identifiées) sont significativement représentées au stade $D$ (ambiance des salles de fabrications) et au stade A (lait de collecte). L'espèce significativement représentée au stade B (lait avant emprésurage) est Debaryomyces hansenii. Kluyveromyces marxianus var. lactis et Candida mogii, forme imparfaite de Zygosaccharomyces rouxii, sont en nombre plus important au stade C (caillé après salage). Enfin, les espèces Kluyveromyces marxianus var. marxianus et Saccharomyces cerevisiae sont surtout présentes à l'intérieur des pâtes fromagères (au stade $\mathrm{KI}$ pour la première et $\mathrm{Jl}$ pour la seconde) tandis que Zygosaccharomyces rouxii, absente au stade $A$, est essentiellement isolée à la surface des fromages (stades JS et KS). Toutes les autres espèces apparaissent indifféremment aux divers stades de prélèvements;

- la prise en compte de la variable "mois" (2), testée par rapport à la variable "espèce", indique que certains mois quelques espèces se détachent de manière significative. Tel est le cas de :

- Kluyveromyces marxianus var. lactis en novembre, mai et juillet, var. marxianus en septembre, var. bulgaricus en février;

- Saccharomyces cerevisiae en novembre (saison de la maturité des pommes) et en août;

- Debaryomyces hansenii en mars et avril;

- le groupe ZYC en janvier, mars, avril et octobre;

- le groupe $X$ en mars, avril et juin.

Il apparaît donc qu'au cours de l'élaboration du fromage de Camembert s'opère une modification importante dans la répartition des espèces de levures. Dans le lait de collecte et dans l'atmosphère de la fro- magerie, il existe une flore composée d'espèces peu ou pas représentées dans les fromages (groupes ZYC et X); lors de la transformation du lait en caillé et surtout après salage, il y a disparition progressive de cette flore, selon un mécanisme qui reste à établir, plusieurs hypothèses pouvant à cet égard être avancées, comme les conditions de milieu $\left(\mathrm{pH}, \mathrm{a}_{\mathrm{w}} . ..\right)$, la nutrition azotée ou carbonée (l'utilisation des lactates notamment)... Par ailleurs, nos résultats mettent clairement en évidence, dans les fromages, la prédominance de l'espèce Kluyveromyces marxianus var. lactis et de sa forme imparfaite Candida sphaerica. Ceci est en accord avec les données de Schmidt et Lenoir (1978, 1980) établies sur le Camembert au lait cru, de Devoyod et Sponem (1970) sur le Roquefort et de Millet et al (1974) sur le Cantal.

\section{CONCLUSION}

Au sein de la flore levure de l'écosystème que représente le fromage de Camembert, on ne trouve qu'un petit nombre d'espèces. La recherche dans les exploitations agricoles des levures susceptibles d'être à l'origine de la "contamination" du lait a permis de mettre en évidence la présence de certaines espèces caractéristiques du fromage, comme Saccharomyces cerevisiae; Kluyveromyces marxianus var. marxianus et Debaryomyces hansenii; en revanche, Kluyveromyces marxianus var. lactis et sa forme imparfaite Candida sphaerica, espèces dominantes fréquemment rencontrées dans les Camemberts, n'ont pas été isolées aux différents points de prélèvements retenus.

Au sein des fromages, il se produit une certaine simplification de la flore levure; en effet, des souches que la méthode simplifiée ne permet pas d'identifier (souches 
des groupes ZYC et $X$ ) sont isolées en assez grand nombre dans le lait de collecte alors qu'elles ne sont présentes que sporadiquement dans les fromages. II apparaît qu'au cours de l'élaboration du fromage il s'exerce un certain effet sélectif sur cette population qui serait sensible à l'action des micro-organismes présents (bactéries, moisissures ou autres espèces de levures) ou aux conditions de milieu imposées par la transformation du lait en fromage (salage, température, source de carbone...). Les autres espèces, isolées du fromage de Camembert, semblent persister, voire même se développer au cours de la fabrication, mais elles le font à des niveaux différents :

- les unes, telles Saccharomyces cerevisiae, Debaryomyces hansenii et Kluyveromyces marxianus var. marxianus sont, le plus souvent, présentes en faibles proportions;

- les autres, comme Kluyveromyces marxianus var. lactis et sa forme imparfaite, prédominent puisqu'elles constituent à chacun des stades de fabrication étudiés environ 50 à $60 \%$ des souches identifiées.

\section{REMERCIEMENTS}

Nous remercions J Lenoir, professeur à l'INA$P G$, directeur du Laboratoire de recherches de la chaire de technologie, pour la lecture critique de ce manuscrit.

Nos remerciements s'adressent également à JJ Daudin (chaire de mathématiques de I'INA$P G)$ pour ses conseils lors du traitement statistique des données.

\section{RÉFÉRENCES}

Barnett JA, Pankhurst RJ (1974) A New Key to the Yeasts. North Holland Publishing Company, Amsterdam, London
Barnett JA, Payne RW, Yarrow D (1979) A Guide to Identifying and Classifying Yeasts. Cambridge University Press, Cambridge

Barnett JA, Payne RW, Yarrow D (1983) Yeasts: Characteristics and Identification. Cambridge University Press, Cambridge

Baroiller C (1988) Mise au point d'une méthode simplifiée d'identification en vue d'une contribution à l'étude de l'origine des levures du fromage de Camembert. Thèse doctorat en sciences, Université de Caen

Baroiller C, Schmidt JL (1984) Mise au point d'une grille simplifiée d'identification des principales espèces de levures présentes dans les fromages. Lait 64, 16-28

Bernhardt E (1946) Time saving in the preparation of corn meal agar in the identification of yeast like fungi. Mycologia 38, 228-229

Brechot P, Chauvet J, Girard H (1962) Identification des levures d'un moût de Beaujolais au cours de sa fermentation. Ann Technol Agric $11,235-244$

Castelli T (1967) Ecologie et systématique des levures de vin. In: Fermentations et vinifications. $2^{\theta}$ Symposium international œnologie Bordeaux-Cognac. INRA, Paris, p 89

Choisy C, Gueguen M, Lenoir J, Schmidt JL, Tourneur $C$ (1987) L'affinage des fromages: les phénomènes microbiens. In: Le Fromage (Eck A, ed.), Tech et Doc Lavoisier, Paris, pp 259-290

Collombier D (1976) Analyse des tables de contingence multidimensionnelles. Ann INSEE 22-23, 55-72

Dale G (1972) Moisissures, levures de la flore du fromage de Saint-Nectaire. Rev Lait Fr 296, 199-203

Devoyod JJ, Sponem D (1970) La flore microbienne du fromage de Roquefort. VI. Les levures. Lait 50, 524-543

Di Menna M (1978) In: The Life of Yeasts. (HJ Phaff, MW Miller, EM Mrak, eds) Harvard University Press, Cambridge, London, pp 217-220

Domercq S (1957a) Etude et classification des levures des vins de la Gironde. Ann Technol Agric 1, 5-58

Domercq S (1957b) Etude et classification des levures des vins de la Gironde. Ann Technol Agric 2, 139-183 
Ducastelle A, Lenoir J (1965) Contribution à l'étude de la flore microbienne du fromage de type Saint-Paulin. II. Ses espèces dominantes. Lait 45, 509-518

Engel G (1986) Hefen in Silagen und Rohmilch. Milchwissenschaft 41, 633-637

Fowell RR (1952) Sodium acetate agar as a sporulation medium for yeasts. Nature 170 , 578

Gripon JC (1978) Levures et moisissures intervenant dans la préparation des produits laitiers. Compte rendu du 20 e Congrès international de laiterie, Paris, 4 ST

Guittonneau G, Keilling J, Delaval H (1939) Les formes levures dans la flore superficielle des fromages de Camembert. Lait 19, 338-353

Hinzelin F, Lectard P (1978) Les levures dans les eaux de la Moselle. Hydrobiologia 61, 209-224

Kreger Van Rij NJW (1984) The Yeasts, a Taxonomic Study. 3rd edition. Elsevier Science Publishers, Amsterdam

Lachance MA, Metcalf BJ, Starmer WT (1982) Yeasts from exudates of Quercus, Ulmus, Populus and Pseudotuga: New isolations and elucidation of some factors affecting ecological specificity. Microb Ecol 8, 191-198

Lenoir J (1962) La flore microbienne du Camembert et son évolution au cours de la maturation. Séances CR Acad Agric Fr 48, 392-399

Lenoir J, Auberger B (1966) Contribution à l'étude de la flore microbienne du fromage de Camembert. Compte rendu du $17 e$ Congrès international de laiterie, Munich, D395-595

Lenoir J, Lamberet G, Schmidt JL (1983) L'élaboration d'un fromage : l'exemple du camembert. Pour Sci 69, 30-42

Lodder J (1971) The Yeasts. A Taxonomic Study. North Holland Publishing Company, Amsterdam, London

Martini A, Federici F, Rosini G (1980) A new approach to the study of yeast ecology of natural substrates. Can J Microbiol 26, 856-859

Michel A, Bizeaud C, Drilleau JF (1988) Flore levurienne présente dans les cidreries de l'ouest de la France. Sci Aliments 8, 359-368

Millet L, Melcion D, Devoyod JJ (1974) La flore microbienne du fromage de Cantal fabriqué à partir de lait cru. III. Rôle des levures dans la fabrication de la "Tome". Lait 54, 616-626

Morris EO (1975) Yeasts from the marine environment. I Appl Bacteriol 38, 211-223

Mrak EM, McClung LS (1940) Yeasts occuring on grapes and products in California. $J$ Bacteriol 40, 395-407

Nunez M, Medina M, Gaya P, Diaz-Amado C (1981) Les levures et les moisissures dans le fromage bleu de Cabrales. Lait 61, 62-79

Park YU (1974) Contribution à l'étude des levures de Cognac. I. Etude et classification des levures de Cognac. Connaiss Vigne Vin $3,253-278$

Peynaud E, Domercq S (1953) Etude des levures de la Gironde. Ann Technol Agric 4, 265-300

Phaff HJ, Miller MW, Mrak EM (1978) In: The Life of Yeasts ( $\mathrm{HJ}$ Phaff, MW Miller, EM Mrak, eds), Harvard University Press, Cambridge, London, pp 200-216, 220-222

Piton C, Richard J (1983) Influence de l'agitation des échantillons de lait cru sur les résultats de dénombrement de trois groupes microbiens d'intérêt industriel. Lait 63, 405-415

Renaud J (1950) Biologie du Vin. Presses Universitaires de France, Paris

Ridgway-Watt P (1979) An improved automatic multipoint inoculator. In: Identification $\mathrm{Me}$ thods for Microbiologists. 2nd, ed. (FA Skinner, DW Lovelock, eds), Academic Press, London, pp 297-304

Saez H (1979) Etude écologique sur les Rhodotorula des homéothermes. Rev Med Vet 130, 903-913

Schmidt JL, Lenoir J (1978) Contribution à l'étude de la flore levure du fromage de $\mathrm{Ca}$ membert. I. Son évolution au cours de la maturation. Lait 58, 355-370

Schmidt JL, Lenoir J (1980) Contribution à l'étude de la flore levure du fromage de $\mathrm{Ca}$ membert. II. Son évolution au cours de la maturation. Lait 60, 275-282

Schmidt JL, Graffard C, Lenoir J (1979) Contribution à l'étude des aptitudes biochimiques de levures isolées du fromage de Camembert. I. Essais préliminaires. Lait 59, 142-163 
Vergeade $J$ (1975) Etude des levures du fromage de Saint-Nectaire. Thèse de doctorat de $3^{\mathrm{e}}$ cycle, Université de Clermont-Ferrand

Wickerham LJ (1946) A critical evaluation of the nitrogen assimilation tests commonly used in the classification of yeasts. J Bacteriol 52, 293-301

Wickerham LJ, Burton KA (1948) Carbon assimilation tests for the classification of yeasts. J Bacteriol 56, 363-371 